\title{
ÚNG DỤNG KỸ THUẬT RAYLEIGH-RITZ TRONG PHÂN TÍCH DAO ĐỘNG TỰ NHIÊN
}

\author{
LÊ VĂN HUN' ${ }^{1}$, ĐỖ CAO PHAN ${ }^{1}$, NGUYỄN HUY CUNG ${ }^{1}$, LÊ HŨU ĐẠT ${ }^{2}$ \\ ${ }^{1}$ Truờng Đại học Công nghiệp thành phố Hồ Chi Minh, \\ ${ }^{2}$ Trường Đại học Giao thông Vận tải;
}

hunglevan@iuh.edu.vn,docaophan@iuh.edu.vn,nguyenhuycung@iuh.edu.vn,lhdat@utc2.edu.vn

Tóm tắt. Bài báo này là một bước trong việc tính toán ứng xử của kết cấu mảnh có mặt cắt phức tạp chịu tác động do gió. Bằng kỹ thuật Rayleigh-Ritz và phương trình Lagrange, bài báo mô tả cách tính dao động riêng của một kêt cấu liên tục dựa trên một hệ thống các phương trình vi phân tuyến tính cấp hai. Phương pháp này sẽ được áp dụng để phân tích dao động riêng của cột ăng ten viễn thông.

Từ khóa.Dao động, cột ăng ten, Rayleigh-Ritz

\section{APPLICATION OF RAYLEIGH-RITZ TECHNIQUE IN ANALYSIS OF NATURAL VIBRATION}

Abstract The paper is the first step towards the aerodynamic analysis of slender structures to wind actions. By Rayleigh-Ritz technique and Lagrange equation, the paper describes the vibrationof a continuous structure though a system of ordinary differential equations in a way more familiar with structural analysts. This vibration method will be applied to antenna pole for dynamic analysis.

Keywords Vibration, antenna pole, Rayleigh-Ritz.

\section{GIÓ́I THIỆ}

Ngày nay, với sự phát triển rất mạnh mẽ của khoa học kỹ thuật, cũng như các yêu cầu từ cuộc sống, Việt Nam đã và đang xây dựng rất nhiều công trình có hình dạng kiến truc đẹp, kết cấu thanh mảnh như các tháp cầu dây văng, dây võng, các dây văng và dây võng, ống khói các nhà máy nhiệt điện, các cột ăng ten viễn thông...

Tuy nhiên, những kết cấu thanh mảnh trên thường kém ổn định dưới tác động của gió. Ứng xử khí động học của chúng trở nên phức tạp và khó kiểm soát hơn với các hiện tượng galloping, flutter,... dẫn đến kết cấu dao động quá biên độ cho phép. Vì vậy, các kết cấu thanh mảnh thường bị phá hủy trong gió bão hoặc không khai thác được trong gió bão như cầu Dubronvik ở Croatia đưa vào sử dụng năm 2002, trong một trận bão năm 2006 đã ghi nhận một cáp dây văng dao động vượt quá biên độ thiết kế. Còn ở Việt Nam đã có rất nhiều cột ăng ten viễn thông bị đổ gẫy trong bão, gây thiệt hại rất lớn về người và của. Bảng 1 là thống kê các tháp ăng tên bị đổ sập trong bão tại Việt Nam trong những năm gần đây.

Bảng 1. Thống kê cột ăng ten đổ trong bão tại Việt Nam [1], [2], [3], [4]

\begin{tabular}{|c|l|c|c|c|}
\hline STT & \multicolumn{1}{|c|}{ Tên công trình } & $\begin{array}{c}\text { Chiều cao } \\
(\mathrm{m})\end{array}$ & Thời gian đổ sập & Nguyên nhân \\
\hline 1 & $\begin{array}{l}\text { Tháp truyền hình Nam Định } \\
\text { (xây dựng năm 2010) }\end{array}$ & 180 & $28 / 10 / 2012$ & $\begin{array}{c}\text { Bão Sơn Tinh (gió } \\
\text { cấp 11) }\end{array}$ \\
\hline 2 & $\begin{array}{l}\text { Tháp ăng ten viến thông tại huyện Đầm Hà, tỉnh } \\
\text { Quảng Ninh }\end{array}$ & 42 & $03 / 08 / 2013$ & $\begin{array}{c}\text { Bão Jebi (gió cấp } 8 \\
\text { cấp 9) }\end{array}$ \\
\hline 3 & $\begin{array}{l}\text { Cột điện đường dây } 500 \mathrm{kV} \text { tại huyện Yên } \\
\text { Dưng, Bắc Giang (xây dựng năm 2015) }\end{array}$ & 30 & $22 / 04 / 2016$ & Mưa lớn và gió lốc \\
\hline 4 & $\begin{array}{l}\text { Gần 10.000 cột điện các loại bị gẫy, nghiêng tại } \\
\text { các tỉnh ven biền miền bắc }\end{array}$ & $27 / 07 / 2016$ & $\begin{array}{c}\text { Bão Mirinae (gió cấp } \\
\text { 8, cấp 9) }\end{array}$ \\
\hline
\end{tabular}




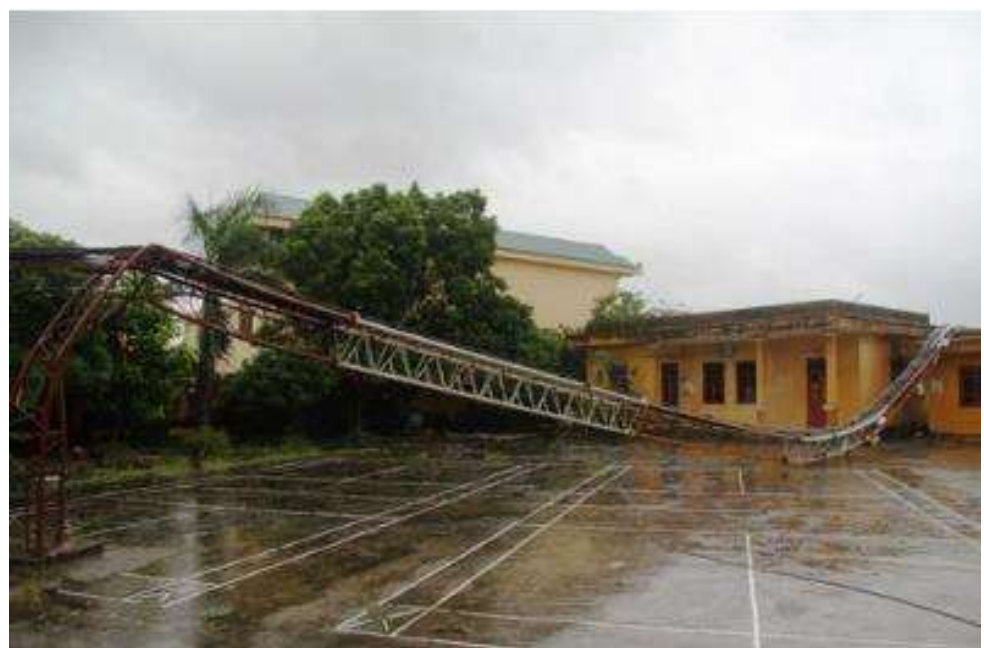

Hinh 1. Cột ăng ten cao hơn 40m của bưu điện huyện Đầm Hà, Quảng Ninh bị đổ trong bão Jebi.[2]

Mặc dù thiệt hại của các công trình trong bão là rất lớn, nhưng tại Việt Nam hiện nay có rất ít công trình nghiên cứu về dao động của kết cấu dưới tác động của gió. Các nghiên cứu ở Việt Nam hiện chỉ dừng lại các đề tài nghiên cứu sinh nghiên cứu về tác động gió lên nhà thấp tầng [5], hay hiện tượng flutter đối với dầm cầu có mặt cắt ngang đối xứng [6]. Trền thế giới, có rất nhiều nhà nghiên cứu nghiên cứu hiện tượng này từ hơn 80 năm trước và tiếp tục cho đến ngày hôm nay, tiêu biểu ở tài liệu tham khảo số [7-10]. Mặc dù các nghiên cứu đã mang lại nhiều thành tựu nhưng bên cạnh đó vẫn còn nhiều hạn chế trong thực tiễ̃n hiện nay.Các nghiên cứu lý thuyết trước đây về ứng xử của kết cấu mảnh do gió thường chỉ xét cho các kết cấu có tiết diện đơn giản. Tuy nhiên, trong thực tế có những kết cấu có mặt cắt rất phức tạp, dẫn đến ứng xử phức tạp của kết cấu do gió. Cột anten là một ví dụ bởi nhiều thành phần như parabol, dây cáp, thang leo ... được gắn vào cột làm cho tiết diện kết cấu phức tạp hơn. Từ đó, các phương pháp giải tích truyển thống để phân tích động cho kết cấu có thể không phù hợp. Để phân tích động của loại kết cấu này, Wahrhaftig và Brasil [11] đã đề xuất phương pháp tính dao động riêng cho cột anten bằng phương pháp xấp xỉ Rayleigh. Tuy nhiên, nghiên cứu đó không bình luận về độ hội tụ của nghiệm và tính chính xác của kết quả phân tích thông qua việc xấp xỉ Rayleigh.

Bài báo này là một bước đầu trong dự án nghiên cứu ứng xử của kết cấu mảnh phức tạp chịu tác động của gió. Trong đó, nghiên cứu tài liệu tham khảo số [12] của dự án này đã đề xuất phương pháp số để xây dựng mô hình tồng quát có thể để phân tích động cho kết cấu mảnh có xét đến độ lệch tâm của tiết diện kết cấu. Ở đó, kỹ thuật Rayleigh-Ritz và phương trình Lagrange được sử dụng để mô tả dao động của kết cấu liên tục bằng một phương trình vi phân thường. Bài báo này áp dụng phương pháp đề xuất ở [12] để phân tích dao động riêng cho một cột anten. Bài báo sử dụng kết cấu này được mô tả ở tài liệu tham khảo số [11] để so sánh kết quả phân tích.

\section{PHÂN TÍCH ĐộNG BẰNG PHƯƠNG PHÁP RAYLEIGH-RITZ}

Phương pháp Rayleigh-Ritz trong việc phân tích động đã được trình bày chi tiết ở tài liệu tham khảo số [12] và được tóm lược ở mục này trong bài báo.

Xét một cấu trúc thanh mảnh có chiều dài $\mathcal{L}$ có mặt cắt ngang chịu gió với vận tốc $U$. Gọi $O x y z$ là hệ tọa Đề các, trong đó gốc $\mathrm{O}$ đặt tại trọng tâm độ cứng của mặt cắt. Các trục $x$ và $y$ là trục nằm ngang, trục $\mathrm{z}$ trùng với trục kết cấu.

Các thành phần chuyển vị do tải trọng gây ra đuợc biểu diễn dưới dạng vector sau như:

$$
\mathbf{r}(z, t)=\left\{\begin{array}{l}
r_{x}(z, t) \\
r_{y}(z, t) \\
r_{z}(z, t)
\end{array}\right\}
$$


trong đó:

$r_{x}$ và $r_{y}$ là chuyển vị thẳng theo phương $x$ và $y$.

$r_{z}$ là chuyển vị xoay quanh trục $\mathrm{z}$.

$t$ là thời gian.

Sử dụng phương pháp Rayleigh-Ritz, mỗi thành phần chuyển vị có thể được tính xấp xỉ như sự tổ hợp tuyến tính như sau:

trong đó:

$$
r_{\alpha}(z, t)=\sum_{k=1}^{N} \phi_{k}^{\alpha}(z) q_{k}^{\alpha}(t)
$$

$q_{k}^{\alpha}(t)$ là hàm chuyển vị tổng quát chưa biết.

$\phi_{k}^{\alpha}(z)$ là hàm dạng.

Thế phương trình (2) vào phương trình (1), chuyển vị cũng có thể viết được dưới dạng ma trận:

$$
\mathbf{r}(z, t)=\Phi(z) \mathbf{Q}(t)
$$

trong đó:

$$
\begin{gathered}
\Phi(z)=\left[\begin{array}{ccc}
\phi_{x}(z) & \emptyset & \emptyset \\
\emptyset & \phi_{y}(z) & \emptyset \\
\emptyset & \emptyset & \phi_{z}(z)
\end{array}\right] \text { và } \phi_{\alpha}(z)=\left[\begin{array}{llll}
\phi_{1}^{\alpha}(z) & \phi_{2}^{\alpha}(z) & \ldots & \phi_{N}^{\alpha}(z)
\end{array}\right] \\
Q(t)=\left\{\begin{array}{l}
\mathbf{Q}_{x}(t) \\
\mathbf{Q}_{y}(t) \\
\mathbf{Q}_{z}(t)
\end{array}\right\} \text { và } \mathbf{Q}_{\alpha}(t)=\left\{\begin{array}{l}
q_{1}^{\alpha}(t) \\
q_{2}^{\alpha}(t) \\
q_{N}^{\alpha}(t)
\end{array}\right\}
\end{gathered}
$$

Từ phương trình (2), ta thấy các hàm dạng $\phi_{k}^{\alpha}(z)$ quyết định đến sự chính xác của phép tính xấp xỉ. Trong khi đó, các chuyển vị tổng quát $q_{k}^{\alpha}(t)$ sẽ được xác định thông qua các phương trình chuyển động. Các phương trình chuyển động này sẽ được xây dựng từ phương trình Lagrange:

$$
\frac{d}{d t}\left[\frac{\partial T(t)}{\partial q_{k}^{\alpha}(t)}\right]-\frac{\partial T(t)}{\partial q_{k}^{\alpha}(t)}+\frac{\partial V(t)}{\partial q_{k}^{\alpha}(t)}=F_{k}^{\alpha}(t)
$$

với: $k=\overline{1, N}, \alpha=x, y, z$

trong đó:

$T$ là động năng,

$V$ là thế năng.

$F_{k}^{\alpha}$ là tổng tải trọng.

Với $T, V$ và $F_{k}^{\alpha}$ được xác định như dưới đây:

$$
\begin{gathered}
T(t)=\sum_{\alpha=x, y, z} T_{\alpha}(t) \\
T_{\alpha}(t)=\frac{1}{2} \int_{(L)}\left[m_{\alpha}(z) \dot{r}_{\alpha}^{2}(z, t)\right] d z \\
V(t)=\sum_{\alpha=x, y, z} V_{\alpha}(t) \\
V_{\alpha}(t)=\frac{1}{2} \int_{(L)} J_{\alpha}\left[\frac{\partial^{2} r_{\alpha}(z, t)}{\partial z^{2}}\right]^{2} d z
\end{gathered}
$$




$$
F_{k}^{\alpha}(t)=\int_{(L)} f_{\alpha}(z, t) \phi_{k}^{\alpha}(z) d z
$$

với:

$m_{x}(z)=m_{y}(z)=m(z)$ là khối lượng trên một đơn vị chiều dài.

$m_{z}(z)=I_{M}(z)$ là mô men quán tính đối với tâm khối lượng $\mathrm{M}$.

$I_{x}=E I_{y}(z)$ và $I_{y}=E I_{x}$ (z)là độ cứng chống uốn.

$I_{z}=G I_{z}(z)$ là độ cứng chống xoắn.

Thay phương trình (7), (9), (11) vào phương trình (6) và một số phép biến đổi toán học, phương trình Lagrange có thể được viết thành:

trong đó:

$$
\mathbf{M Q ̈}(t)+\mathbf{K Q}(t)=\mathbf{F}(t)
$$

$$
\begin{aligned}
\mathbf{M} & =\left[\begin{array}{ccc}
\mathbf{M}_{x} & \emptyset & \emptyset \\
\emptyset & \mathbf{M}_{y} & \emptyset \\
\emptyset & \emptyset & \mathbf{M}_{z}
\end{array}\right] \text { và } \mathbf{K}=\left[\begin{array}{ccc}
\mathbf{K}_{x} & \emptyset & \emptyset \\
\emptyset & \mathbf{K}_{y} & \emptyset \\
\emptyset & \emptyset & \mathbf{K}_{z}
\end{array}\right] \\
\mathbf{F}(t) & =\left\{\begin{array}{l}
\mathbf{F}_{x}(t) \\
\mathbf{F}_{y}(t) \\
\mathbf{F}_{z}(t)
\end{array}\right\} \mathbf{F}_{\alpha}(t)=\left[\begin{array}{llll}
F_{1}^{\alpha}(t) & F_{2}^{\alpha}(t) & \ldots & F_{N}^{\alpha}(t)
\end{array}\right]^{T}
\end{aligned}
$$

Phần tử (i,j) trong ma trận $\mathbf{M}_{\alpha}, \mathbf{M}_{\alpha \theta}, \mathbf{K}_{\alpha}$ của phương trình (13) được cho bởi

$$
\left(\mathbf{K}_{\alpha}\right)_{i j}=k_{i j}^{\alpha},\left(\mathbf{K}_{\alpha}\right)_{i j}=k_{i j}^{\alpha}
$$

trong đó:

$$
\begin{gathered}
m_{i j}^{\alpha}=\int_{(L)} m_{\alpha}(z) \phi_{i}^{\alpha}(z) \phi_{j}^{\alpha}(z) d z \\
m_{i j}^{x z}=k_{i j}^{\alpha}=\int_{(L)} J_{\alpha}(z) \frac{d^{2} \phi_{i}^{\alpha}(z)}{d z^{2}} \frac{d^{2} \phi_{j}^{\alpha}(z)}{d z^{2}} d z
\end{gathered}
$$

Cuối cùng, liên quan đến ma trận giảm chấn $\mathrm{C}$, phương trình chuyển động đầy đủ ba chiều có thể được viết như sau:

$$
\mathbf{M Q ̈}(t)+\mathbf{C Q}(t)+\mathbf{K Q}(t)=\mathbf{F}(t)
$$

Như vậy, nhờ sự xấp xỉ Rayleigh-Ritz và phương trình Lagrange, sự chuyển động của một kết cấu liên tục được điều khiển bởi một hệ phương trình vi phân tuyến tính cấp 2 (ODE) như trong phương trình (18) thay vì phương trình vi phân từng phần (PDE).Đây là một hệ có vô số bậc tự do giảm xuống hệ có $3 \mathrm{~N}$ bậc tự do. Tuy nhiên, các đặc trưng kết cấu vẫn được giữ nguyên, chẳng hạn như sự liên tục của hình dạng hay chuyển vị mà không xuất hiện ở nhiều bậc tự do. Đây là một ưu điểm khi phân tích động học của hệ thông qua hệ ODE trong phương trình (18), về cơ bản là quen thuộc và dễ dàng với kỹ sư hơn PDE. Sự chính xác của phương pháp này đã được chứng minh và thảo luận ở tài liệu tham khảo số [12].

\section{VÍ DU SỐ}

Cột angten cao 30m được mô tả ở tài liệu tham khảo số [11] được dùng để phân tích các đặc trưng động học theo phương pháp đã trình bày ở mục 2 . Kết quả tính toán tần số dao động được lấy từ chương trình matlab và phần mềm Midas civil và so sánh với kết quả từ tài liệu tham khảo số [11].

Kết cấu cột ăng ten trong tính toán ví dụ là: Cột ang ten hình trụ rỗng cao $30 \mathrm{~m}$, có đường kính ngoài ở đáy và đỉnh lần lượt là $82 \mathrm{~cm}$ và $52 \mathrm{~cm}$ như hình 2 . Dọc theo chiều cao cột có gắn có chảo ăng ten tại các vị trí: dàn ăng ten I nặng $880 \mathrm{~kg}$ ở đỉnh cột, dàn ăng ten II nặng $228 \mathrm{~kg}$ ở độ cao $27 \mathrm{~m}$ và dàn ăng ten 
III nặng $924 \mathrm{~kg}$ ở độ cao $20 \mathrm{~m}$. Đường kính ngoài $\Phi_{\text {ext }}$ và chiều dày $\mathrm{t}$ thay đổi theo độ cao theo Bảng 2 với các thông số tính toán đặc trưng của vật liệu và khối lượng tác dụng lên cột ăng ten ở Bảng 3.

Tác giả lập trình matlab để tính dao động của cột ăng ten theo lý thuyết ở mục 2 của bài báo này.

Để so sánh kết quả tính dao động theo lý thuyết ở mục 2 của bài báo này, ngoài kết quả từ tài liệu tham khảo số [11], tác giả mô phỏng tính toán dao động của kết cấu bằng phần mềm Midas civil. Cột ăng ten được mô hình hóa là một cột có một đầu ngàm, một đầu tự do gồm 33 phần tử, chịu khối lượng tập trung tại các nút tương ứng với các độ cao $20 \mathrm{~m}, 27 \mathrm{~m}$ và $30 \mathrm{~m}$ với mô hình tính toán như hình $2 \mathrm{c}$.

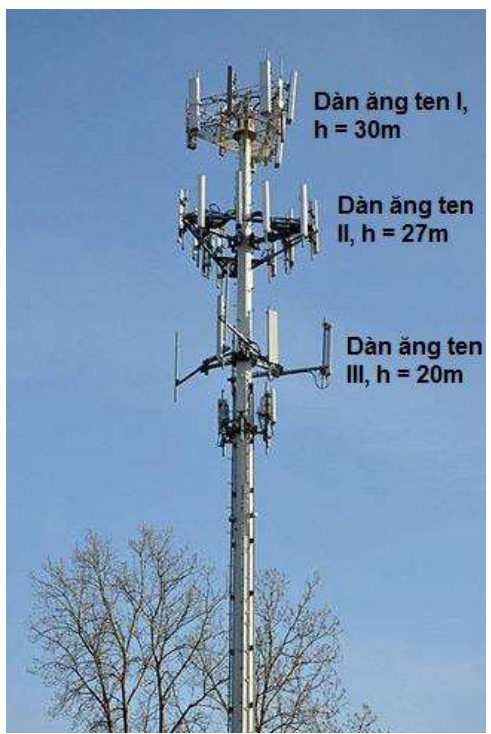

(a) Kết cấu cột ăng ten ngoài thực tế

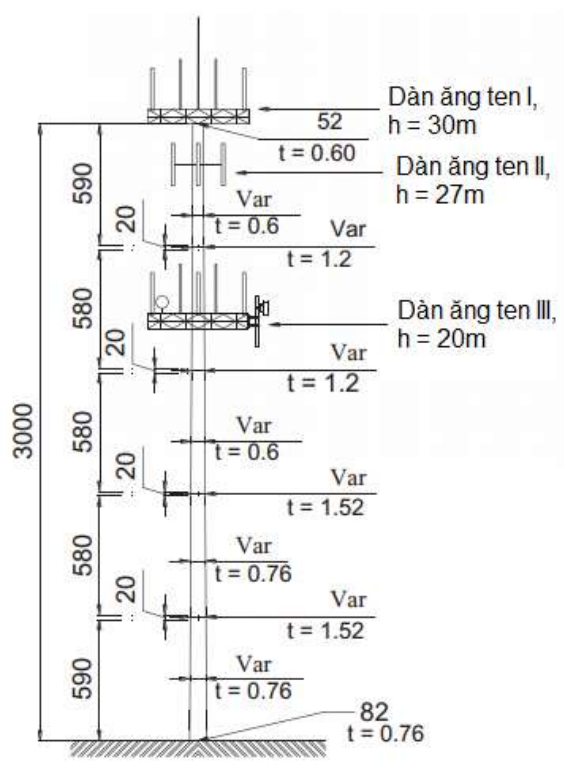

(b) Mô hình 2D cột ăng ten

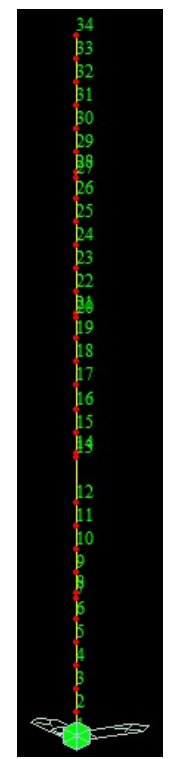

(c) Mô hình 2D cột ăng ten trên Midas

Hinh 2. Sơ đồ cấu tạo cột ăng ten $30 \mathrm{~m}$

Bảng 2. Sơ đồ cấu tạo cột ăng ten $30 \mathrm{~m}$

\begin{tabular}{|c|c|c|c|c|c|c|c|c|}
\hline $\mathrm{h}(\mathrm{m})$ & $\Phi_{\text {ext }}(\mathrm{cm})$ & $\mathrm{t}(\mathrm{cm})$ & $\mathrm{h}(\mathrm{m})$ & $\Phi_{\text {ext }}(\mathrm{cm})$ & $\mathrm{t}(\mathrm{cm})$ & $\mathrm{h}(\mathrm{m})$ & $\Phi_{\text {ext }}(\mathrm{cm})$ & $\mathrm{t}(\mathrm{cm})$ \\
\hline 30,0 & 52,0 & 0,6 & 20,0 & 62,0 & 0,6 & 10,0 & 72,0 & 0,76 \\
\hline 29,0 & 53,0 & 0,6 & 19,0 & 63,0 & 0,6 & 9,0 & 73,0 & 0,76 \\
\hline 28,0 & 54,0 & 0,6 & 18,1 & 63,9 & 0,6 & 8,0 & 74,0 & 0,76 \\
\hline 27,0 & 55,0 & 0,6 & 17,9 & 64,1 & 0,6 & 7,0 & 75,0 & 0,76 \\
\hline 26,0 & 56,0 & 0,6 & 17,0 & 65,0 & 0,6 & 6,1 & 75,9 & 0,76 \\
\hline 25,0 & 57,0 & 0,6 & 16,0 & 66,0 & 0,6 & 5,9 & 76,1 & 0,76 \\
\hline 24,1 & 57,9 & 0,6 & 15,0 & 67,0 & 0,6 & 5,0 & 77,0 & 0,76 \\
\hline 23,9 & 58,1 & 0,6 & 14,0 & 68,0 & 0,6 & 4,0 & 78,0 & 0,76 \\
\hline 23,0 & 59,0 & 0,6 & 13,0 & 69,0 & 0,6 & 3,0 & 79,0 & 0,76 \\
\hline 22,0 & 60,0 & 0,6 & 12,1 & 69,9 & 0,6 & 2,0 & 80,0 & 0,76 \\
\hline 21,0 & 61,0 & 0,6 & 11,9 & 70,1 & 0,76 & 1,0 & 81,0 & 0,76 \\
\hline & & & & & & 0,0 & 82,0 & 0,76 \\
\hline
\end{tabular}


Bảng 3. Các thông số tính toán cho cột ăng ten $30 \mathrm{~m}$

\begin{tabular}{|l|c|c|}
\hline \multicolumn{1}{|c|}{ Thông số } & Giá trị & Đơn vị \\
\hline Mô đun đàn hồi của thép, $\mathrm{E}$ & 205 & $\mathrm{Gpa}$ \\
\hline Khối lượng riêng của thép, $\rho$ & 7850 & $\mathrm{~kg} / \mathrm{m}^{3}$ \\
\hline Khối lượng tập trung ở đỉnh ăng ten (dàn ăng ten I) & 880 & $\mathrm{~kg}$ \\
\hline Khối lượng tập trung ở độ cao 27m (dàn ăng ten II) & 228 & $\mathrm{~kg}$ \\
\hline Khối lượng tập trung ở độ cao 20m (dàn ăng ten III) & 924 & $\mathrm{~kg}$ \\
\hline Khối lượng phân bố trên một đơn vị chiều dài & 40 & $\mathrm{~kg} / \mathrm{m}$ \\
\hline Gia tốc trọng trường, $g$ & 9,81 & $\mathrm{~m} / \mathrm{s}^{2}$ \\
\hline
\end{tabular}

Tần số dao dộng của cột ang ten được thể hiện ở Bảng 4.

Bảng 4. Tần số dao động của cột ăng ten $30 \mathrm{~m}$

\begin{tabular}{|l|c|c|}
\hline & Tần số dao động $(\mathrm{Hz})$ & Sai số so với [11] (\%) \\
\hline Theo $[11]$ & 0,550 & --- \\
\hline Theo Midas civil & 0,547 & 0,55 \\
\hline Theo lý thuyết trình bày ở mục 2 của bài báo & 0,470 & 14,55 \\
\hline
\end{tabular}

Từ Bảng 4 ta thấy: sử dụng phương pháp đã trình bày ở mục 2, tần số dao động cơ bản của cột ăng ten $30 \mathrm{~m}$ là $0,47 \mathrm{~Hz}$. Trong đó, các hàm dạng được sử dụng trong tính toán là các hàm đa thức bậc cao và cho kết quả hội tụ tốt trong khoảng từ $0,47 \mathrm{~Hz}$ đến $0,55 \mathrm{~Hz}$.

Kết quả tính toán theo tài liệu tham khảo số [11] của tần số dao động cột ăng ten $30 \mathrm{~m}$ là $0,55 \mathrm{~Hz}$ (sai số $0,56 \%$ ) và từ phần mềm Midas là $0,547 \mathrm{~Hz}$ (sai số $0,55 \%$ ). Như vậy, dùng phương pháp Rayleigh để tính xấp xỉ chuyển vị trong tài liệu tham khảo số [11] cho kết quả tương tự với phương pháp phần tử hữu hạn.

Sự khác biệt trong kết quả tính toán của phương pháp Rayleigh và phương pháp phần tử hữu hạn với xấp xỉ Rayleigh-Ritz là $15 \%$. Nguyên nhân có thể từ việc sử dụng hàm dạng. Do tài liệu tham khảo số [11] và phần mềm Midas (phương pháp phần tử hữu hạn) chỉ sử dụng 1 hàm dạng lượng giác để xấp xỉ chuyển vị và không nhắc tới tính hội tụ của nghiệm xấp xỉ khi chỉ sử dụng 1 hàm dạng. Vì vậy, kết quả từ bài báo này đáng tin cậy hơn.

\section{KẾT LUẬN}

Bài báo đã áp dụng một phương pháp hiệu quả để phân tích động cho kết cấu mảnh. Bằng phương pháp Rayleigh-Ritz, phương trình dao động được biểu diễn dưới dạng tuyến tính bậc 2 . Phương pháp được áp dụng để xác định đặc trưng động học của một cột angten thực có tiết diện phức tạp. Kết quả phân tích đáng tin cậy hơn phương pháp của các tác giả khác.

Phương pháp phân tích trong bài báo này là một bước trong việc phân tích ứng xử của kết cấu mảnh chịu tác động do gió. Từ phương trình dao động đã được xây dựng sẽ cho phép thiết lập phương pháp tính thuận tiên hơn bài toán bất ổn định của kết cẩu do gió với các kết cấu có mặt cắt phức tạp. Việc phân tính bất ổn định này sẽ được trình bày trong các bài báo tiếp theo.

\section{LỜI CẢM ƠN}

Các tác giả cảm ơn "Chương trình Vườn ươm sáng tạo khoa học và công nghệ trẻ” - Trung tâm phát triển khoa học và công nghệ trẻ - Thành đoàn thành phố Hồ Chí Minh đã hỗ trợ kinh phí nghiên cứu thông qua đề tài: "Phân tích ứng xử khí động học của kết cấu thanh mảnh nhạy cảm với gió" năm 2017. 


\section{TÀI LIỆU THAM KHẢO}

[1] http://www.baogiaothong.vn/can-canh-5-thap-phat-thanh-truyen-hinh-do-sap-do-bao-d65973.html.

[2] http://vnexpress.net/tin-tuc/thoi-su/chua-ghi-nhan-thiet-hai-ve-nguoi-do-bao-jebi-2859946.html

[3] http://nld.com.vn/thoi-su-trong-nuoc/cot-dien-500-kv-bi-giat-do-mat-dien-dien-rong-20160423084552976.htm

[4] http://vtv.vn/chuyen-dong-24h/thai-binh-nhieu-cot-dien-gay-do-sau-bao-so-1-do-chua-du-tieu-chuan20160727101035557.htm

[5] N.H. Nam, Nghiên cứu giải pháp giảm áp lực gió lên mái dốc nhà thấp tầng bằng thực nghiệm trong ống thổi khí động, Luận án tiến sĩ, Viện khoa học công nghệ xây dụng, Bộ xây dựng, 2014.

[6] N.V. Mỹ, Nghiên cứu biện pháp nâng cao ổn định khí động học flutter trong kết cấu cầu hệ treo, Luận án tiến sĩ, Trường đại học Xây dụng, 2015.

[7] Desai, Y. M., Shah, A. H., and Popplewell, N., Galloping-analysis for two-degree-of-freedom oscillator, $J$. Engrg. Mech., ASCE, 116(12),1990, 2583-2602.

[8] Jones, K. F.,Coupled vertical and horizontal galloping. J. Engrg. Mech., ASCE, 118(1), 1992, $92-107$.

[9] J.P.D. Hartog,Transmission-line vibration due to sleet, Institute of Electrical Engineers, 51, 1932, $1074-1086$.

[10] Martin, Wallace W., Currie, I.G., Naudascher, Eduard. Streamwise oscillations of cylinders, J. Eng. Mech. Division-ASCE, 107(3),1981, 589-607.

[11] Alexandrede M.Wahrhaftig, Reyolando M.L.R.F. Brasil, Vibration analysis of mobile phone mast system by Rayleigh method, Appiled Mathematical Modelling, 2016, 1-16.

[12] Nguyen C.H, Numerical modelling the aeroelastic response of irregular slender structures, The International conference on advances in Computational Mechanics Acome 2017, August 02-04, Phu Quoc, Vietnam, 2017.

Ngày nhận bài: 29/09/2017

Ngày chấp nhận đăng: 31/12/2017 